\title{
From autoimmune hepatitis to $Q$ fever
}

\author{
Davide Tizzani, Silvia Totaro, Valentina Laura Crudo, Andrea Viola, Vittorio Gallo, Franco Veglio \\ Department of Medical Sciences, University of Torino, Città della Salute e della Scienza di Torino Hospital, Torino, Italy
}

\begin{abstract}
Q fever is an infectious disease caused by Coxiella burnetii. Its clinical presentation is often nonspecific and the serological diagnosis difficult to make, especially in the absence of specific and suspected medical history. This article presents a case of fever of unknown origin (FUO), interpreted as an autoimmune hepatitis, later proven by the liver biopsy to be a granulomatous hepatitis caused by C. burnetii. The approach to FUO, the features of granulomatous hepatitis and Q fever are presented and discussed.
\end{abstract}

\section{Case Report}

L-V. M. is a 42-year-old man with unknown medical history except for obesity and an excessive consumption of alcohol and derived. He has come to our attention for a persistent fever, higher than $38^{\circ} \mathrm{C}$, with chills, mainly but not exclusively in the evening, that lasted for more than 3 weeks. It was not associated with specific organ symptoms and in his past and recent medical history there were no clues to suspect a specific etiology, according to the most recent literature. ${ }^{1}$ No consumption of any drugs, over-the-counter products or narcotic substances were reported.

Previously investigated at another hospital, the fever was associated with significant and persistent in-

Correspondence: Davide Tizzani, Department of Medical Sciences, University of Torino, Città della Salute e della Scienza di Torino Hospital, via Genova 3, 10126 Torino, Italy. Mobile: +39.334.3254163 - Fax: +39.011.6336931.

E-mail: davidet2001@yahoo.it

Key words: Fever of unknown origin; granulomatous hepatitis; Q fever.

Contributions: TD, TS and VA wrote the text; CVL checked the English form; VG and VF reviewed the manuscript.

Conflict of interest: the authors declare no potential conflict of interest.

Received for publication: 22 October 2014.

Revision received: 29 January 2015.

Accepted for publication: 5 February 2015.

This work is licensed under a Creative Commons Attribution NonCommercial 4.0 License (CC BY-NC 4.0).

CCopyright D. Tizzani et al., 2016

Licensee PAGEPress, Italy

Italian Journal of Medicine 2016; 10:58-61

doi:10.4081/itjm.2015.556 creases in enzymes of hepatic cytolisis and cholestasis (Table 1); serological investigations proved positive exclusively for rheumatoid factor (RF) and antismooth muscle antibodies (ASMA). The fever was responsive to a first antibiotic course with ceftriaxone (7 days) and to a following second antibiotic course with piperacillin-tazobactam ( 5 days), administered in two previous admissions. In both situations, there was a complete resolution of the fever followed by a new recurrence of the symptoms. The abdominal ultrasonography and abdominal magnetic resonance imaging/magnetic resonance cholangiography showed a picture of hepatic steatosis, without further morphological alterations.

In our division, we confirmed the known defects of hepatic cytolisis and cholestasis; we found a significant increase in C-reactive protein in the absence of leukocytosis and of procalcitonin increase. Lymphocytes immunophenotyping was negative. Regarding autoimmunity, we found a positive low title for RF, lupus anticoagulant and anticardiolipin antibodies. We broadened our serological investigations with the observation of positive low title for Widal Wright reaction. The standard cultures (three sets of blood culture, urine culture, culture of sputum with special search for mycobacterium and oropharyngeal swab) were all negative. A chest X-ray and an echocardiogram were negative for pleuro-parenchymal lesions and for endocardial vegetations or masses. Our final diagnostic test was a liver biopsy.

While waiting for the histological result, we started steroid therapy (dosage $1 \mathrm{mg} / \mathrm{kg}$ ) in the suspect of an autoimmune disease. We got a full and stable defervescence, but only a partial reduction in hepatic enzymes of cytolisis and cholestasis and in C-reactive protein (Table 1). After two weeks we got the result of liver biopsy that reported the presence of steatosis with a severe lipogranulomatosis, with the indications to carry out further investigations in the setting of acquired diseases, above all infectious diseases.

We performed additional microbiological tests, in- 
cluding the specific serological investigations for Q fever (immunofluorescence assay using Coxiella burnetii antigen), which proved a recent infection by $C$. burnetii. Therefore, therapy with doxycicline was started, with simultaneous tapering and then suspension of steroid therapy. We observed a complete resolution of fever and a full normalization of the hepatic enzymes of cytolisis and cholestasis and of the markers of inflammation (Table 1). The serological test was repeated 4 weeks later with a significant rise (four fold) in antibody titers.

\section{Discussion}

The classic fever of unknown origin (FUO) is a diagnostic challenge for the clinician. According to a recent review of the literature, about $25 \%$ of the cases of FUO remains without a precise diagnosis. ${ }^{1}$

The aforementioned clinical case displays a classic FUO, with the presence of a potential diagnostic clue (the laboratory abnormalities of the liver enzymes) ${ }^{2}$ that has induced the application of a target-oriented diagnostic algorithm, as suggested by international literature for the management of FUO, ${ }^{3}$ and the execution of a liver biopsy, which in literature has proved to be a good diagnostic yield. ${ }^{4}$ In any case, these proposed flow charts do not include the serological investigations of $\mathrm{Q}$ fever in the absence of a suggestive medical history, mainly the contact with animals. ${ }^{1,4}$ Nevertheless the implementation of this test is necessary in the presence of other suspicious data.

The biopsy picture showed a nonspecific granulomatous hepatitis. The granulomas, focal accumulation of epithelioid cells (modified macrophages), often associated with other inflammatory cells, are a nonspecific reaction of the immune system to different etiologic agents, systemic or primarily in liver or idiopathic. This finding is present in $2.4-15 \%$ of all liver biopsies, with a greater frequency in patients in close examination for FUO. ${ }^{5}$ The most frequent causes are sarcoidosis, mycobacterium infections, primary biliary cirrhosis, cancers, drugs-induced damage. ${ }^{6}$

The morphology of granuloma is often not specific enough in order to identify the causative agent, but it can help in the differential diagnostic examination, as well as the presence of necrosis, the location within the liver parenchyma and the cellular infiltrate accompanying the granuloma.

In our patient the situation was complicated by the presence of a double granulomatous hepatitis: next to lipogranulomas (histiocytes and macrophages around a core of triglycerides), typical of hepatic steatosis, and compatible with patient's medical history epithelioid granulomas were also present; in the latter, the macrophages differentiate in secretory cells with homogeneous cytoplasm with no inclusion bodies, but with deposition of fibrin and collagen around the granuloma (fibrin-ring granuloma). This ring is rather suggestive but not diagnostic of $\mathrm{Q}$ fever because it is also present in many other infectious diseases (leishmaniasis, rickettsia infection, Staphylococcus epidermidis, salmonella, cytomegalovirus, Epstein-Barr virus, hepatitis A virus, mycobacterial infection, typhoid fever, toxoplasmosis); it can also be caused by drugs (allopurinol) or it may be associated with autoimmune diseases (systemic lupus erythematosus, Horton arteritis) or cancers (Hodgkin lymphoma).$^{7-9}$

Therefore the sole examination and morphological description of the granuloma is never sufficient to reach a conclusive diagnosis, for which the combination of clinical and laboratory data is essential.

In the presence of FUO and liver epithelioid granuloma proved at histological examination, the execution of extended serological investigations is necessary and consequential, including those for hepatitis viruses, syphilis, brucella, rickettsia, leishmaniasis and coxiella.

Our patient showed a serological profile compatible with an acute infection by $C$. burnetii.

C. burnetii is an intracellular bacterium responsible for Q fever, a zoonosis spread all over the world.

The flu-like clinical features of this infection are highly unspecific and make it difficult to suspect and to identify (Table 2)..$^{10}$

The incidence is very low and so this infection is considered a rare disease, but given the nonspecific clinical picture there is a heavy bias of underestimation.

In Italy the incidence, influenced by a low clinical suspicion, is very low: ${ }^{11}$ in the years 2001 and 2002, respectively only 739 and 769 infections by C. burnetii were reported, while the serological prevalence was found to be $3.8 \%$ in general population and $73.4 \%$ in groups at risk of infection. ${ }^{12}$

Table 1. Levels of hepatic cytolisis and cholestasis enzyme at different times.

\begin{tabular}{lccc}
\hline & $\begin{array}{c}\text { At admission } \\
\text { to Hospital }\end{array}$ & $\begin{array}{c}\text { After steroidal therapy-at start } \\
\text { of therapy with doxycycline }\end{array}$ & $\begin{array}{c}\text { At end of therapy } \\
\text { with doxycycline }\end{array}$ \\
\hline AST/ALT (UI/L) & $201 / 304$ & $80 / 120$ & $31 / 33$ \\
\hline GGT/ALP (UI/L) & $610 / 163$ & $402 / 147$ & $36 / 54$ \\
\hline
\end{tabular}

AST, aspartate transaminase; ALT, alanine aminotransferase; GGT, gamma-glutamyl transferase; ALP, alkaline phosphatase. 
The human infection is usually determined by inhalation of environmental dust contaminated by $C$. burnetii, which is excreted in milk, urine and feces of infected animals (cattle, sheep and goats) and is shed in high numbers within the amniotic fluids and the placenta during birth or by direct contact with these materials. Tick bites, ingestion of unpasteurized milk or dairy products, human-to-human transmission are rare ways of infection. However all these data were silent in the clinical history of our patient. Similarly, he did not live in a rural area or near a farm. However, the international literature reports cases of infection or outbreaks where there was no clear exposure: ${ }^{13}$ in fact infectious elements can persist in the environment for months and they can be carried away by the wind. ${ }^{10}$

The infection occurs, in most cases, like a flu syndrome; in $85 \%$ of cases, there can be also lung or liver involvement, often only laboratory and not clinical involvement (as in our patient) (Table 2).

Therefore, after ruling out the main viral, infectious and immunological causes, a liver involvement should raise the suspicion of infection by $C$. burnetii, also in the absence of overt risk factors. In our clinical case, there were some confounding factors, such as the antibody positivity for RF, ASMA, antiphospholipid and the positive low title for Widal Wright reaction: the literature reports this cross reactivity in the infection by C. burnetii. ${ }^{14}$

Table 2. Percentage of acute $Q$ fever patients with selected clinical findings.

\begin{tabular}{lc}
\hline \multicolumn{2}{c}{ Clinical finding of acute Q fever } \\
\hline Clinical finding & \% of patients \\
\hline Fever & $88-100$ \\
\hline Fatigue & $97-100$ \\
\hline Chills & $68-88$ \\
\hline Headache & $68-98$ \\
\hline Myalgia & $47-69$ \\
\hline Sweats & $31-98$ \\
\hline Cough & $24-90$ \\
\hline Nausea & $22-49$ \\
\hline Vomiting & $13-42$ \\
\hline Chest pain & $10-45$ \\
\hline Diarrhea & $5-22$ \\
\hline Skin rash & $5-21$ \\
\hline Myocarditis & $0.5-1$ \\
\hline Pericarditis & 1 \\
\hline Meningoencephalitis & $1-2$ \\
\hline Meath & \\
\hline
\end{tabular}

Modified from Maurin and Raoult $1999^{10}$ with permission.
According to the latest international recommendations, a compatible clinical presentation, the positive serology and the subsequent serological trend are all necessary to the diagnosis of $\mathrm{Q}$ fever. In our clinical case we did not perform the search of DNA by polymerase chain reaction, considered gold standard in literature, but we relied for a correct diagnosis on the execution and correct interpretation of liver biopsy.

Therefore, we have to suspect the infection by $C$. burnetti and have to perform the serological investigations in presence of FUO and compatible clinical features, even in the absence of work-related risk factors or a clear contact with animals.

After a successful 14-days therapy with doxycycline, the patient is at present in follow up for the assessment of development of chronic Q fever. This follow up is very important because $C$. burnetii has the ability to persist for long time in the host and in $5 \%$ of the patients the disease could become chronic, soon (within 6 weeks) or later (also some years), causing particularly a severe endocarditis $(60-70 \%$ of all reported cases) and, less frequently, aortic aneurysms and infection of the bone, liver or reproductive organs.

\section{Conclusions}

The diagnostic flow chart of FUO should be modified according to the results of investigations in progress, considering also serological exams previously not performed.

The liver biopsy is certainly to be considered in the presence of FUO with increases in liver enzymes, after excluding the main viral, infectious and immunological causes.

The granulomatous hepatitis is a nonspecific histological picture, which must be related to the clinical picture in order to get to a specific etiological diagnosis.

$\mathrm{Q}$ fever is an infrequent but expanding infection in Italy, which we must think of as possible responsible of a fever of unknown origin, mostly in the presence of alterations of liver enzymes, even in the absence of suspected history and clear risk factors.

\section{References}

1. Hayakawa K, Ramasamy B, Chandrasekar PH. Fever of unknown origin: an evidence-based review. Am J Med Sci 2012;344:307-16.

2. Bleeker-Rovers CP, Vos FJ, de Kleijn EM, et al. A prospective multicenter study on fever of unknown origin: the yield of a structured diagnostic protocol. Medicine (Baltimore) 2007;86:26-38.

3. Gaeta GB, Fusco FM, Nardiello S. Fever of unknown origin: a systematic review of the literature for 19952004. Nucl Med Commun 2006;27:205-11. 
4. Mourad O, Palda V, Detsky AS. A comprehensive evidence-based approach to fever of unknown origin. Arch Inter Med 2003;163:545-51.

5. Coash M, Forouhar F, Wu CH, Wu GY. Granulomatous liver diseases: a review. J Formos Med Assoc 2012; 111:3-13.

6. Wainwright H. Hepatic granulomas. Eur J Gastroenterol Hepatol 2007;19:93-5.

7. Kleiner DE. Granulomas in the liver. Semin Diagn Pathol 2006;23:161-9.

8. Lamps LW. Hepatic granulomas, with an emphasis on infectious causes. Adv Anat Pathol 2008;15:309-18.

9. Font J, Bruguera M, Perez-Villa F, Ingelmo M. Hepatic fibrin-ring granulomas caused by Staphylococcus epidermidis generalized infection. Gastroenterology 1987; 93:1449-51.
10. Maurin M, Raoult D. Q fever. Clin Microbiol Rev 1999; 12:518-53.

11. Santoro D, Giura R, Colombo MC, et al. Q fever in Como, Northern Italy. Emerg Infect Dis 2004;10:159-60.

12. Monno R, Fumarola L, Trerotoli P, et al. Seroprevalence of Q fever, brucellosis and leptospirosis in farmers and agricultural workers in Bari, southern Italy. Ann Agric Environ Med 2009;16:205-9.

13. Anderson A, Bijlmer H, Fournier PE, et al. Diagnosis and management of Q fever - United States, 2013. Recommendations from $\mathrm{CDC}$ and the Q Fever Working Group. MMWR Recomm Rep 2013;62:1-30.

14. Holmes RO Jr, Hartzell JD, Tofferi JK, et al. Dual high titer antineutrophil cytoplasmic autoantibodies in association with systemic Q fever. J Clin Rheumatol 2009;15:411-3. 